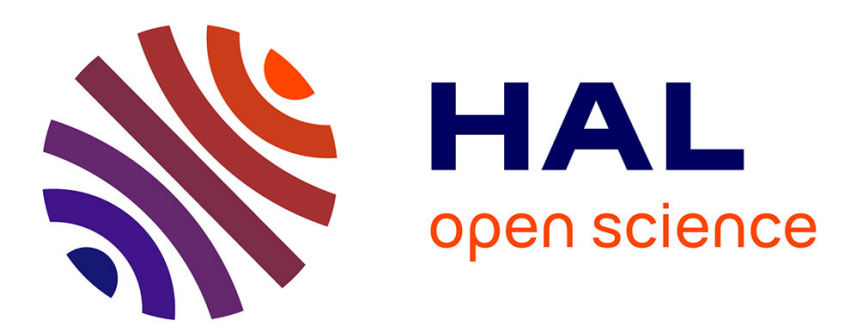

\title{
Relative Similarity Logics are Decidable: Reduction to FO2 with Equality
}

\author{
Stéphane Demri, Beata Konikowska
}

\section{To cite this version:}

Stéphane Demri, Beata Konikowska. Relative Similarity Logics are Decidable: Reduction to FO2 with Equality. European Workshop on Logics in Artificial Intelligence (JELIA'98), Jurgen Dix; Luis Farinas del Cerro; Ulrich Furbach, Oct 1998, Dagstuhl, Germany. pp.279-293, 10.1007/3-540-49545-2_19 . hal-03195324

\section{HAL Id: hal-03195324 \\ https://hal.science/hal-03195324}

Submitted on 11 Apr 2021

HAL is a multi-disciplinary open access archive for the deposit and dissemination of scientific research documents, whether they are published or not. The documents may come from teaching and research institutions in France or abroad, or from public or private research centers.
L'archive ouverte pluridisciplinaire HAL, est destinée au dépôt et à la diffusion de documents scientifiques de niveau recherche, publiés ou non, émanant des établissements d'enseignement et de recherche français ou étrangers, des laboratoires publics ou privés. 


\title{
Relative Similarity Logics are Decidable: Reduction to FO $^{2}$ with Equality*
}

\author{
Stéphane Demri and Beata Konikowska \\ 1 Laboratoire LEIBNIZ - C.N.R.S. \\ 46 av. Félix Viallet, 38000 Grenoble, France \\ 2 Institute of Computer Science, Polish Academy of Sciences \\ Ordona 21, 01-237 Warszawa, Poland
}

\begin{abstract}
We show the decidability of the satisfiability problem for relative similarity logics that allow classification of objects in presence of incomplete information. As a side-effect, we obtain a finite model property for such similarity logics. The proof technique consists of reductions into the satisfiability problem for the decidable fragment $\mathrm{FO}^{2}$ with equality from classical logic. Although the reductions stem from the standard translation from modal logic into classical logic, our original approach (for instance handling nominals for atomic properties and decomposition in terms of components encoded in the reduction) can be generalized to a larger class of relative logics, opening ground for further investigations.
\end{abstract}

\section{Introduction}

Background. Classification of objects in presence of incomplete information has been long recognized as an issue of concern for various AI problems that deal with commonsense knowledge as well as scientific and engineering knowledge (expert systems, image recognition, knowledge bases and so on). Similarity -sometimes termed "weak equivalence"- provides a basic tool each time when we classify objects with respect to their properties. There exist several formal systems capturing the notion of similarity from the logical viewpoint [Vak91a, Vak91b]. In the present paper we base on the formalization given in [Kon97], where, contrary to [Vak91a, Vak91b], similarity is treated as a relative notion. More precisely, in [Kon97] similarity is defined as a reflexive and symmetric binary relation $\operatorname{sim}_{P}$, parametrized by the set $P$ of properties with respect to which the objects are classified as either similar or dissimilar. Thus, instead of a single similarity relation we have a whole family $\left(\operatorname{sim}_{P}\right)_{P \subset P R O P}$, where $P R O P$ is the set of all the properties considered in a given system. When talking about similarity or equivalence it is natural to talk about lower and upper approximation $L\left(\operatorname{sim}_{P}\right) A$, $U\left(\operatorname{sim}_{P}\right) A$ of a given set $A$ of objects with respect to the similarity $\operatorname{sim}_{P}$. The above operations stem from rough set theory [Paw81], with $L\left(\operatorname{sim}_{P}\right) A$ being the set of all objects in $A$ which are not similar (in the sense of $\operatorname{sim}_{P}$ ) to any

\footnotetext{
* This work has been partially supported by the Polish-French Project "Rough-set based reasoning with incomplete information: some aspects of mechanization”, $\sharp 7004$.
} 
object outside $A$ and $U\left(\operatorname{sim}_{P}\right) A$ - the set of all objects of the universe which are similar to some object in $A$. Thus, the above operations could be considered as the operations of taking "interior" and "closure" of the set $A$ with respect to similarity $\operatorname{sim}_{P}$. However, the analogy is not complete, since similarity is not transitive, and hence the above operations are not idempotent.

Practical importance of the approximation operations is quite obvious: if we can distinguish objects only up to similarity, then when looking for objects belonging to some set $A$ we should take those in $L\left(\operatorname{sim}_{P}\right) A$, if we want to consider only the objects sure to belong to $A$, and those in $U\left(\operatorname{sim}_{P}\right) A$ if our aim is not to overlook any object which might possibly belong to $A$.

Our objectives. The formal system introduced in [Kon97] features the above operations, which generate a family of interdependent relative modalities. The resulting polymodal logic is equipped with a complete deduction system. However, from the viewpoint of any practical applications of the similarity logic in the area of Artificial Intelligence mentioned above an issue of great importance is whether the logic is decidable. A positive answer to this question might provide not only a decision procedure, but also a better understanding of the logical analysis of similarity. These are the objectives of the present paper. Up to now, the question of decidability has been open, which is hardly surprising in view of the high expressive power of the logic. Indeed: its language admits implicitly the universal modal operator, and nominals for atomic propositions as well for atomic properties; in addition, the modal operators are interdependent. Nominals (or names) are used in numerous non-classical logics with various motivations (see e.g. [Orło84a, PT91, Bla93, Kon97]) and they usually greatly increase the expressive power of the logics (causing additional difficulties with proving (un)decidability -see e.g. [PT91]). Furthermore, since finite submodels can be captured in the language up to an isomorphism (which is yet another evidence of the expressive power of similarity logics), there is no hope of proving decidability by showing a finite model property for a class of models including strictly the class of standard models with a bound on the model's size (see e.g. [Vak91c, Bal97]). On the other hand, the intersection operator, which is implicitly present in the interpretation of the modal terms, is known to behave badly for filtration-like constructions.

Our contribution. We prove that the logic defined in [Kon97] together with some of its variants is decidable by translating it to a decidable fragment of firstorder logic: the two-variable fragment $\mathrm{FO}^{2}$ containing equality, but no function symbols (see e.g. [Mor75]). Although there are known methods of handling the universal modal operator, the Boolean operations for modal terms and nominals for atomic propositions in order to translate them into $\mathrm{FO}^{2}$ with equality (see for example the survey papers [Ben98, Var97]), the extra features of the similarity logics require some significant extra work in order to be also translated to such a fragment. This is achieved in the present paper. Unlike the Boolean Modal Logic BML [GP90], for which decidability can be proved via the finite model property for a class of models, reduction of satisfiability for the similarity logics 
to $\mathrm{FO}^{2}$ with equality is the only known decidability proof we are aware of, and therefore we solve an open problem here. As a side-effect, we prove the finite model property. More importantly, the novelty of our approach allows us to generalize the translation to a large class of relative modal logics.

Plan of the paper. The paper is structured as follows. In Section 2 the relative similarity logics we deal with in the paper are defined, and some results about their expressive power and complexity are stated. In Section 3, we define the translation of the main relative similarity logic $\mathcal{L}$ into $\mathrm{FO}^{2}$ with equality, and show its faithfulness. Decidability and finite model property for $\mathcal{L}$ are obtained partly by considering the analogous properties of the fragment $\mathrm{FO}^{2}$ with equality. In Section 4, we investigate some variants of $\mathcal{L}$, and show their decidability and the finite model property. Section 5 concludes the paper by providing some generalizations of the results proved in the preceding Sections, and stating what is known about the computational complexity of $\mathcal{L}$-satisfiability. In addition, several examples of formula translations are given.

\section{Similarity logics}

\subsection{Information systems and similarity}

The information systems that proposed for representation of knowledge are the foundational structures, on which the semantics of the relative similarity logic is based. An information system $S$ is defined as a pair $\langle E N T, P R O P\rangle$ where ENT is a non-empty set of entities (also called objects) and PROP is a nonempty set of properties (also called attributes) -see e.g. [Paw81]. Each property prop is a mapping $E N T \rightarrow \mathcal{P}\left(V_{\text {al }} l_{\text {prop }}\right) \backslash \emptyset$ and $V a l_{\text {prop }}$ is the set of values of the property prop -see e.g. [OP84]. In that setting, two entities $e_{1}, e_{2}$ are said to be similar with respect to some set $P \subseteq P R O P$ of properties (in short $\left.e_{1} \operatorname{sim}_{P} e_{2}\right)$ iff for any prop $\in P$, prop $\left(e_{1}\right) \cap \operatorname{prop}\left(e_{2}\right) \neq \emptyset$. The polymodal frames of the relative similarity logics are isomorphic to structures of the form $\left(E N T, P R O P,\left(\operatorname{sim}_{P}\right)_{P \subset P R O P}\right)$. Other relationships between entities can be found in the literature -see e.g. [FdCO84, Orto84b]. For instance, two entities $e_{1}, e_{2}$ are said to be negatively similar (resp. indiscernible) with respect to some set $P \subset P R O P$ of properties (in short $e_{1}$ nsim $_{P} e_{2}$-resp. $e_{1}$ ind $d_{P} e_{2}$ ) iff for any prop $\in P,-\operatorname{prop}\left(e_{1}\right) \cap-\operatorname{prop}\left(e_{2}\right) \neq \emptyset$ - resp. $\operatorname{prop}\left(e_{1}\right)=\operatorname{prop}\left(e_{2}\right)$.

The family $\left(\operatorname{sim}_{P}\right)_{P \subset P R O P}$ of similarity relations stemming from some information system $S=\langle E \bar{N} T, P R O P\rangle$ induces certain approximations of subsets of entities in $S$. Indeed, let $L\left(\operatorname{sim}_{P}\right) X$ (resp. $\left.U\left(\operatorname{sim}_{P}\right) X\right)$ be the lower (resp. upper) $\operatorname{sim}_{P}$-approximation of the set $X$ of entities defined as follows:

- L $\left(\operatorname{sim}_{P}\right) X \stackrel{\text { def }}{=}\left\{e \in E N T: \forall e^{\prime} \in E N T,\left(e, e^{\prime}\right) \in \operatorname{sim}_{P}\right.$ implies $\left.e^{\prime} \in X\right\}$;

$-U\left(\operatorname{sim}_{P}\right) X \stackrel{\text { def }}{=}\left\{e \in E N T: \exists e^{\prime} \in E N T,\left(e, e^{\prime}\right) \in \operatorname{sim}_{P}\right.$ and $\left.e^{\prime} \in X\right\}$.

Obviously $L\left(\operatorname{sim}_{P}\right) X \subseteq X \subseteq U\left(\operatorname{sim}_{P}\right) X$ and $L\left(\operatorname{sim}_{P}\right) X=E N T \backslash U\left(\operatorname{sim}_{P}\right)(E N T \backslash$ $X)$. These approximations are rather crucial in rough set theory since they allow to classify objects in presence of incomplete information. That is why, the 
semantics of modal operators in the relative similarity logics shall use these approximations as modal operations. We invite the reader to consult [Oe97] for examples of rough set analysis of incomplete information.

\subsection{Syntax and semantics}

The set of primitive symbols of the polymodal language $\mathrm{L}$ is composed of

- a set VARSE $=\left\{E_{1}, E_{2}, \ldots\right\}$ of variables representing sets of entities,

- a set VARE $=\left\{\mathrm{x}_{1}, \mathrm{x}_{2}, \ldots\right\}$ of variables representing individual entities,

- symbols for the classical connectives $\neg, \wedge$ (negation and conjunction), and

- a countably infinite set $\{[\mathrm{A}]: \mathrm{A} \in \mathrm{TERM}\}$ of unary modal operators where the set TERM of terms is the smallest set containing

- the constant 0 representing the empty set of properties,

- a countably infinite set $\operatorname{VARP}=\left\{\mathrm{p}_{1}, \mathrm{p}_{2}, \ldots\right\}$ of variables representing individual properties,

- a countably infinite set VARSP $=\left\{\mathrm{P}_{1}, \mathrm{P}_{2}, \ldots\right\}$ of variables representing sets of properties,

and closed under the Boolean operators $\cap, \cup,-$.

The formation rules of the set FORM of formulae are those of the classical propositional calculus plus the rule: if $F \in$ FORM and $A \in$ TERM, then $[A] F \in$ FORM. We use the connectives $\vee, \Rightarrow, \Leftrightarrow,\langle\mathrm{A}\rangle$ as abbreviations with their standard meanings. For any syntactic category $\mathrm{X}$ and any syntactic object 0 , we write $\mathrm{X}(0)$ to denote the set of those elements of $\mathrm{X}$ that occur in 0 . Moreover, for any syntactic object 0 , we write $\mid$ 이 to denote its length (or size), that is the number of symbol occurrences in 0 . As usual, $s u b(\mathrm{~F})$ denotes the set of subformulae of the formula $\mathrm{F}$ (including $\mathrm{F}$ itself).

Definition 1. A TERM-interpretation $v$ is a map $v:$ TERM $\rightarrow \mathcal{P}(P R O P)$ such that $P R O P$ is a non-empty set and for any $\mathrm{A}_{1}, \mathrm{~A}_{2} \in \mathrm{TERM}$,

- if $\mathrm{A}_{1}, \mathrm{~A}_{2} \in$ VARP and $\mathrm{A}_{1} \neq \mathrm{A}_{2}$, then $v\left(\mathrm{~A}_{1}\right) \neq v\left(\mathrm{~A}_{2}\right)$,

- if $\mathrm{A}_{1} \in$ VARP, then $v\left(\mathrm{~A}_{1}\right)$ is a singleton, i.e. $v\left(\mathrm{~A}_{1}\right)=\{$ prop $\}$ for some prop $\in$ $P R O P$,

$-v(0)=\emptyset, v\left(\mathrm{~A}_{1} \cap \mathrm{A}_{2}\right)=v\left(\mathrm{~A}_{1}\right) \cap v\left(\mathrm{~A}_{2}\right), v\left(\mathrm{~A}_{1} \cup \mathrm{A}_{2}\right)=v\left(\mathrm{~A}_{1}\right) \cup v\left(\mathrm{~A}_{2}\right)$,

$-v\left(-\mathrm{A}_{1}\right)=P R O P \backslash v\left(\mathrm{~A}_{1}\right)$.

For any $A, B \in$ TERM, we write $A \equiv 0$ (resp. $A \equiv B$ ) when for any TERMinterpretation $v, v(\mathrm{~A})=\emptyset(\operatorname{resp} . v(\mathrm{~A})=v(\mathrm{~B}))$.

Definition 2. A model $\mathcal{U}$ is a structure $\mathcal{U}=\left(E N T, P R O P,\left(\operatorname{sim}_{P}\right)_{P \subseteq P R O P}, v\right)$ where ENT and $P R O P$ are non-empty sets and $\left(\operatorname{sim}_{P}\right)_{P \subseteq P R O P}$ is a family of binary relations over ENT such that

- for any $\emptyset \neq P \subseteq P R O P$, $\operatorname{sim}_{P}$ is reflexive and symmetric,

- for any $P, P^{\prime} \subseteq P R O P, \operatorname{sim}_{P \cup P^{\prime}}=\operatorname{sim}_{P} \cap \operatorname{sim}_{P^{\prime}}$ and $\operatorname{sim}_{\emptyset}=E N T \times E N T$. 
Moreover, $v$ is a mapping $v:$ VARE $\cup$ VARSE $\cup$ TERM $\rightarrow \mathcal{P}(E N T) \cup \mathcal{P}(P R O P)$ such that $v(\mathrm{E}) \subseteq E N T$ for any $\mathrm{E} \in \mathrm{VARSE}, v(\mathbf{x})=\{e\}$, where $e \in E N T$ for any $\mathrm{x} \in \mathrm{VARE}$ and the restriction of $v$ to TERM is a TERM-interpretation.

Since the set of nominals for properties is countably infinite, and any two different nominals are interpreted by different properties, each model has an infinite set of properties. Let $\mathcal{U}=\left(E N T, P R O P,\left(\operatorname{sim}_{P}\right)_{P \subset P R O P}, v\right)$ be a model. As usual, we say that a formula $\mathrm{F}$ is satisfied by an entity $e \in E N T$ in $\mathcal{U}$ (written $\mathcal{U}, e \vDash \mathrm{F}$ ) if the following conditions are satisfied.

$-\mathcal{U}, e \models \mathrm{x}$ iff $\{e\}=v(\mathrm{x}) ; \mathcal{U}, e \models \mathrm{E}$ iff $e \in v(\mathrm{E}) ;$

$-\mathcal{U}, e \models \neg \mathrm{F}$ iff not $\mathcal{U}, e \models \mathrm{F} ; \mathcal{U}, e \models \mathrm{F} \wedge \mathrm{G}$ iff $\mathcal{U}, e \models \mathrm{F}$ and $\mathcal{U}, e \models \mathrm{G}$;

$-\mathcal{U}, e \models[\mathrm{A}] \mathrm{F}$ iff for any $e^{\prime} \in \operatorname{sim}_{v(\mathrm{~A})}(e), \mathcal{U}, e^{\prime} \models \mathrm{F}$.

A formula $\mathrm{F}$ is true in a model $\mathcal{U}$ (written $\mathcal{U} \models \mathrm{F}$ ) iff for any $e \in E N T, \mathcal{U}, e \models$ $\mathrm{F}$ - or, equivalently, iff for some $e \in E N T, \mathcal{U}, e \models[0] \mathrm{F}$. A formula $\mathrm{F}$ is said to be valid iff $F$ is true in all models. A formula $F$ is said to be satisfiable iff $\neg F$ is not valid. The similarity logic $\mathcal{L}$ is said to have the finite model propertyiff every satisfiable formula is satisfied in some model $\mathcal{U}=\left(E N T, P R O P,\left(\operatorname{sim}_{P}\right)_{P \subset P R O P}, v\right)$ with a finite set $E N T$ such that, for any $P \subseteq P R O P, \operatorname{sim}_{P}=\operatorname{sim}_{P \cap P_{0}}$, where $P_{0} \subset P R O P$ is finite and nonempty ( $P_{0}$ is called the relevant part of $P R O P$ in $\overline{\mathcal{U}})$. Consequently, if $\mathcal{L}$ has the finite model property, then every satisfiable formula has a model $\left(E N T, P R O P,\left(\operatorname{sim}_{P}\right)_{P \subset P R O P}, v\right)$ such that for any $\emptyset \neq P \subseteq P R O P, \operatorname{sim}_{P}=\cap_{x \in P} \operatorname{sim}_{\{x\}}$.

The similarity logic defined in [Kon97] is not exactly the logic $\mathcal{L}$ defined above, since in [Kon97] the set of properties was supposed to be fixed, and constants representing properties were used instead of variables. For any set $X$, we write $\mathcal{L}_{X}$ to denote the logic that differs from $\mathcal{L}$ in the following points: (1) the set of properties PROP is fixed in all the models and equals $X,(2)$ VARP and $X$ have the same cardinality. In various places in the paper, we implicitly use the facts that satisfiability is insensitive to the renaming of any sort of variables, and that any two models isomorphic in the standard sense satisfy the same set of formulae. Moreover, for the logics $\mathcal{L}_{X}$, as far as satisfiability is concerned, it is irrelevant whether we fix the interpretation of each nominal for the properties.

\subsection{Expressive power and complexity lower bound}

Since the language of the relative similarity logic $\mathcal{L}$ contains nominals, the universal modal operator and a family of standard modal operators, its expressive power is quite high. In Proposition 3 below, we shall state a counterpart of Corollary 4.17 in [GG93] (see also Theorem 2.8 in [PT91]) saying that finite submodels can be captured in the language up to isomorphism. In Proposition 3 below, we show that for any finite structure $\mathcal{S}$ there is a formula $F_{\mathcal{S}}$ such that a model $\mathcal{U}$ satisfies $\mathrm{F}_{\mathcal{S}}$ iff $\mathcal{S}$ is a substructure of $\mathcal{U}$ up to isomorphism. Although this shows that the expressive power of the logic is high, it has a very unpleasant consequence: there is no hope of characterizing $\mathcal{L}$-satisfiability by a class of finite non-standard models the way it is done in [Vak91c, Bal97]. It means for instance 
that proving the finite model property of $\mathcal{L}$ by a standard filtration-like technique becomes highly improbable since $\mathcal{L}$ has implicitly the intersection operator in the language.

In Proposition 3 below, the structure $\mathcal{S}$ encodes a finite part of some model. The set $\{1, \ldots, n\}$ should be understood as a finite set of entities, and $\{1, \ldots, l\}$ as a finite set of properties. Moreover, only a finite set $\left\{\mathrm{E}_{1}, \ldots, \mathrm{E}_{k}\right\}$ of atomic propositions is taken into account. For $i \in\{1, \ldots, n\}$ and $j \in\{1, \ldots, k\}, j \in v^{\prime}(i)$ is to mean that $\mathrm{E}_{j}$ is satisfied by $i$.

Proposition 3. Let $\mathcal{S}=\left\langle\{1, \ldots, n\},\{1, \ldots, l\},(R(P))_{P \subseteq\{1, \ldots, l\}}, v^{\prime}\right\rangle$ be a structure such that each $R(P)$ is a reflexive and symmetric relation, $R(\emptyset)$ is the universal relation, for any $P, P^{\prime} \subseteq\{1, \ldots, l\}, R\left(P \cup P^{\prime}\right)=R(P) \cap R\left(P^{\prime}\right)$ and $v^{\prime}$ is a mapping $\{1, \ldots, n\} \rightarrow \mathcal{P}(\{1, \ldots, k\})$ for some $k \geq 1$. Then, there is formula $\mathrm{F}_{\mathcal{S}}$ such that for any $\mathcal{L}$-model $\mathcal{U}, \mathcal{U} \models \mathrm{F}_{\mathcal{S}}$ iff there is an $1-1$ mapping $\Psi_{1}:\{1, \ldots, n\} \rightarrow$ ENT and an injective mapping $\Psi_{2}:\{1, \ldots, l\} \rightarrow$ PROP with the following properties

- for any $i \in\{1, \ldots, k\}, v\left(\mathrm{E}_{i}\right)=\left\{\Psi_{1}(s): i \in v^{\prime}(s)\right\}$;

- for any $P \subseteq P R O P$ such that there is $P^{\prime} \subseteq\{1, \ldots, l\}$ verifying $\left\{\Psi_{2}(i): i \in\right.$ $\left.P^{\prime}\right\}=P$, we have $\operatorname{sim}_{P}=R\left(P^{\prime}\right)$.

Proof. The formula $\mathrm{F}_{\mathcal{S}}$ is the conjunction of the following formulae.

1. $[0]\left(\mathrm{x}_{1} \vee \ldots \vee \mathrm{x}_{n}\right) \Leftrightarrow[0] \bigwedge_{1 \leq i<j \leq n} \neg\left(\mathrm{x}_{i} \wedge \mathrm{x}_{j}\right)$;

2. $[0]\left(\bigwedge_{i \in\{1, \ldots, n\}}\left(\mathrm{x}_{i} \Rightarrow\left(\bigwedge_{u \in\{1, \ldots, k\}} s_{u} \mathrm{E}_{u}\right)\right)\right)$ where $s_{u}$ is the empty string if $u \in$ $v(i)$, otherwise $s_{u} \stackrel{\text { def }}{=} \neg$;

3. for any $\left\{i_{1}, \ldots, i_{q}\right\} \subseteq\{1, \ldots, l\}$ and all $i \in\{1, \ldots, n\}$,

$$
[0]\left(\mathrm{x}_{i} \Rightarrow\left(\left(\bigwedge_{j \in R_{\left\{i_{1}, \ldots, i_{q}\right\}}(i)}\left\langle\mathrm{p}_{i_{1}} \cup \ldots \cup \mathrm{p}_{i_{q}}\right\rangle \mathrm{x}_{j}\right) \wedge\left(\bigwedge_{j \notin R_{\left\{i_{1}, \ldots, i_{q}\right\}}(i)} \neg\left\langle\mathrm{p}_{i_{1}} \cup \ldots \cup \mathrm{p}_{i_{q}}\right\rangle \mathrm{x}_{j}\right)\right)\right.
$$

Before establishing decidability of $\mathcal{L}$-satisfiability, one can provide a lower bound for the complexity of this problem using [Hem96].

Proposition 4. $\mathcal{L}$-satisfiability is EXPTIME-hard.

When no nominals for properties and entities are allowed satisfiability can be shown to be in EXPTIME [Dem98].

\section{Translation from $\mathcal{L}$ into $\mathrm{FO}^{2}$ with equality}

\subsection{A known decidable fragment of classical logic}

Consistently with the general convention, by $\mathrm{FO}^{2}$ we mean a fragment of firstorder logic (FOL for short) without equality or function symbols using only 2 variables (denoted by $\mathrm{y}_{0}$ and $\mathrm{y}_{1}$ in the sequel). We shall translate the similarity logics into a slight extension of $\mathrm{FO}^{2}$ obtained by augmenting the language with identity. Actually, we shall restrict ourselves to the following vocabulary: 
- a countable set $\left\{\mathrm{P}_{i}: i \in \omega\right\} \cup\left\{\mathrm{Q}_{i}: i \in \omega\right\}$ of unary predicate symbols,

- a countable set $\left\{\mathrm{R}_{i, j}: i, j \in \omega\right\}$ of binary predicate symbols,

- the symbol $=($ interpreted as identity $)$.

In what follows, by a first-order formula we mean a formula belonging to just this fragment of FOL (written $\mathrm{FO}^{2}[=]$ in the sequel). As usual, a first-order structure $\mathcal{M}$ (restricted to this fragment) is a pair $\langle D, m\rangle$ such that $D$ is a non-empty set and $m$ is an interpretation function with $m\left(\mathrm{P}_{i}\right) \cup m\left(\mathbf{Q}_{i}\right) \subseteq D$ for $i \in \omega$, $m\left(\mathrm{R}_{i, j}\right) \subseteq D \times D$ for $i, j \in \omega$ and $m(=) \stackrel{\text { def }}{=}\{\langle a, a\rangle: a \in D\}$. As usual, a valuation $v_{\mathcal{M}}$ for $\overline{\mathcal{M}}$ is a mapping $v_{\mathcal{M}}:\left\{\mathrm{y}_{0}, \mathrm{y}_{1}\right\} \rightarrow D$. We write $\mathcal{M}, v_{\mathcal{M}} \vDash \mathrm{F}$ to denote that $\mathrm{F}$ is satisfied in $\mathcal{M}$ under $v_{\mathcal{M}}$, and omit $v_{\mathcal{M}}$ when $\mathrm{F}$ is closed. It is known that $\mathrm{FO}^{2}[=]$ has the finite model property, $\mathrm{FO}^{2}[=]$-satisfiability is decidable [Mor75] and NEXPTIME-complete [Lew80, GKV97]. Actually, F is $\mathrm{FO}^{2}[=]$-satisfiable iff $F$ has a model of size $2^{c \times|F|}$ for some fixed $c>0$ [GKV97].

\subsection{Normal forms}

Let $\mathrm{F} \in$ FORM be such that ${ }^{3} \operatorname{VARP}(\mathrm{F})=\left\{\mathrm{p}_{1}, \ldots, \mathrm{p}_{l}\right\}$ and $\operatorname{VARSP}(\mathrm{F})=\left\{\mathrm{P}_{1}, \ldots, \mathrm{P}_{n}\right\}$. In the rest of this section, we assume that $n \geq 1$ and $l \geq 1$. The degenerate cases make no additional difficulties and they are treated in a separate section. For any integer $k \in\left\{0, \ldots, 2^{n}-1\right\}$, by $\mathrm{B}_{k}$ we denote the term

$$
\mathrm{A}_{1} \cap \ldots \cap \mathrm{A}_{n}
$$

where, for any $s \in\{1, \ldots, n\}, \mathrm{A}_{s}=\mathrm{P}_{s}$ if $b_{i t}(k)=0$, and $\mathrm{A}_{s}=-\mathrm{P}_{s}$ otherwise, with bit $_{s}(k)$ denoting the $s$ th bit in the binary representation of $k$. For any integer $k \in\left\{0, \ldots, 2^{n}-1\right\}$, we denote

$$
\mathrm{A}_{k, 0} \stackrel{\text { def }}{=} \mathrm{B}_{k} \cap-\mathrm{p}_{1} \cap \ldots \cap-\mathrm{p}_{l}
$$

Finally, for any $\left\langle k, k^{\prime}\right\rangle \in\left\{0, \ldots, 2^{n}-1\right\} \times\{1, \ldots, l\}$, we denote $\mathrm{A}_{k, k^{\prime}} \stackrel{\text { def }}{=} \mathrm{B}_{k} \cap \mathrm{p}_{k^{\prime}}$. For any TERM-interpretation $v:$ TERM $\rightarrow \mathcal{P}(P R O P)$, the family

$$
\left\{v\left(\mathrm{~A}_{k, k^{\prime}}\right):\left\langle k, k^{\prime}\right\rangle \in\left\{0, \ldots, 2^{n}-1\right\} \times\{0, \ldots, l\}\right\}
$$

is a partition of $P R O P$. Moreover, for any term $\mathrm{A} \in \mathrm{TERM}(\mathrm{F})$, either $\mathrm{A} \equiv 0$ or there is a unique non-empty set $\left\{A_{k_{1}, k_{1}^{\prime}}, \ldots, A_{k_{u}, k_{u}^{\prime}}\right\}$ such that $A \equiv A_{k_{1}, k_{1}^{\prime}} \cup$ $\ldots \cup \mathrm{A}_{k_{u}, k_{u}^{\prime}}$. The normal form of $\mathrm{A}$, written $N(\mathrm{~A})$, is either 0 or $\mathrm{A}_{k_{1}, k_{1}^{\prime}} \cup \ldots \cup$ $\mathrm{A}_{k_{u}, k_{u}^{\prime}}$ according to the two cases above. Such a decomposition, introduced in [Kon97], generalizes with nominals the canonical disjunctive normal form for the propositional calculus. $N(\mathrm{~A})$ can be computed by an effective procedure.

For any $k^{\prime} \in\{1, \ldots, l\}$, we write $o c_{k}$, to denote the set

$$
\left\{k \in\left\{0, \ldots, 2^{n}-1\right\}: \exists \mathrm{A} \in \operatorname{TERM}(\mathrm{F}), N(\mathrm{~A})=\ldots \cup \mathrm{A}_{k}, k^{\prime} \cup \ldots\right\}
$$

\footnotetext{
${ }^{3}$ Without any loss of generality we can assume that if $l$ (resp. $n$ ) nominals for properties (resp. for entities) occur in $\mathrm{F}$ they are precisely the $l$ (resp. $n$ ) first in the enumeration of VARP (resp. VARE) since satisfiability is not sensitive to the renaming of variables.
} 
Informally, occ $k^{\prime}$ is the set of indices $k$ such that $\mathrm{A}_{k, k^{\prime}}$ occurs in the normal form of some element of TERM(F). We write setocc $_{k^{\prime}}$ to denote the set

$$
\left\{X \subseteq \operatorname{occ}_{k^{\prime}}: \operatorname{card}\left(\operatorname{occ}_{k^{\prime}}\right)-1 \leq \operatorname{card}(X) \leq 2^{n}-1\right\}
$$

The definition of setocc $_{k^{\prime}}$ is motivated by the fact that for any TERM-interpretation $v$, there is only one $k \in\left\{0, \ldots, 2^{n}-1\right\}$ such that $v\left(\mathrm{~A}_{k}, k^{\prime}\right) \neq \emptyset$, and for this very $k, v\left(\mathrm{~A}_{k, k^{\prime}}\right)=v\left(\mathrm{p}_{k^{\prime}}\right)$. For each $X \in$ setocc $_{k^{\prime}}$ in turn, in the forthcoming constructions we shall enforce $v\left(\mathrm{~A}_{k, k^{\prime}}\right)=\emptyset$ for any $k \in X$.

\subsection{The translation}

In this section, we define an extension to $\mathcal{L}$ of the translation $S T$ defined in [Ben83] of modal formulae into a first-order language containing a binary predicate, a countable set of unary predicate symbols and two individual variables (due to a smart recycling of the variables). Our translation of the nominals for entities is similar to the translation of nominals in [GG93]. However, we take into account the decomposition of terms into components in order to obtain a faithful translation. The translation of nominals for atomic properties is a twofold one: we take it into account both in defining the normal form of terms, and in the generalized disjunction defining the translation $\mathrm{T}$ below.

Let $\mathrm{F} \in$ FORM be such that $\operatorname{VARP}(\mathrm{F})=\left\{\mathrm{p}_{1}, \ldots, \mathrm{p}_{l}\right\}, \operatorname{VARSP}(\mathrm{F})=\left\{\mathrm{P}_{1}, \ldots, \mathrm{P}_{n}\right\}$ and $\operatorname{VARE}(\mathrm{F})=\left\{\mathrm{x}_{1}, \ldots, \mathrm{x}_{q}\right\}$. Before defining $S T^{\prime}$ - the mapping translating $\mathcal{L}$ formulae into $\mathrm{FO}^{2}$-formulae - let us state what are the main features we intend that mapping to have. Analogously to $S T, S T^{\prime}$ encodes the quantification in the interpretation of $[\mathrm{A}]$ into the language of $\mathrm{FO}^{2}$ by using the standard universal quantifier $\forall$ and by introducing a binary predicate symbol $\mathrm{R}_{\mathrm{A}}$ for each $\mathrm{A} \in \mathrm{TERM}$. However, this is not exactly the way $S T^{\prime}$ is defined. Actually, to each component $\mathrm{A}_{k, k^{\prime}}$ we associate the predicate symbol $\mathrm{R}_{k}, k^{\prime}$. The main idea of $S T^{\prime}$ is therefore to treat components as constants, which means that the translation of $[\mathrm{A}] \mathrm{G}$ is uniquely determined by the components (if any) of the normal form of A. Then, the conditions on the $\mathcal{L}$-models justify why a modal operator indexed by the union of components is translated into a formula involving a conjunction of atomic formulae. Let $S T^{\prime}$ be defined as follows ( $S T^{\prime}$ is actually parametrized by F and $i \in\{0,1\})$ :

(1) $S T^{\prime}\left(\mathrm{E}_{j}, \mathrm{y}_{i}\right) \stackrel{\text { def }}{=} \mathrm{P}_{j}\left(\mathrm{y}_{i}\right) ; S T^{\prime}\left(\mathrm{x}_{j}, \mathrm{y}_{i}\right) \stackrel{\text { def }}{=} \mathrm{Q}_{j}\left(\mathrm{y}_{i}\right)$;

(2) $S T^{\prime}\left(\neg \mathrm{G}, \mathrm{y}_{i}\right) \stackrel{\text { def }}{=} \neg S T^{\prime}\left(\mathrm{G}, \mathrm{y}_{i}\right) ; S T^{\prime}\left(\mathrm{G} \wedge \mathrm{H}, \mathrm{y}_{i}\right) \stackrel{\text { def }}{=} S T^{\prime}\left(\mathrm{G}, \mathrm{y}_{i}\right) \wedge S T^{\prime}\left(\mathrm{H}, \mathrm{y}_{i}\right)$;

(3)

$$
S T^{\prime}\left([\mathrm{A}] \mathrm{G}, \mathrm{y}_{i}\right) \stackrel{\text { def }}{=}\left\{\begin{array}{l}
\forall \mathrm{y}_{0} S T^{\prime}\left(\mathrm{G}, \mathrm{y}_{0}\right) \text { if } N(\mathrm{~A})=0 \\
\forall \mathrm{y}_{1-i}\left(\mathrm{R}_{k_{1}, k_{1}^{\prime}}\left(\mathrm{y}_{i}, \mathrm{y}_{1-i}\right) \wedge \ldots \wedge \mathrm{R}_{k_{u}, k_{u}^{\prime}}\left(\mathrm{y}_{i}, \mathrm{y}_{1-i}\right)\right) \Rightarrow S T^{\prime}\left(\mathrm{G}, \mathrm{y}_{1-i}\right) \\
\text { if } N(\mathrm{~A})=\mathrm{A}_{k_{1}, k_{1}^{\prime}} \cup \ldots \cup \mathrm{A}_{k_{u}, k_{u}^{\prime}}
\end{array}\right.
$$

By adopting the standard definition $\langle\mathrm{A}\rangle \mathrm{G} \stackrel{\text { def }}{=} \neg[\mathrm{A}] \neg \mathrm{G}, S T^{\prime}$ can be easily defined for $\langle A\rangle$ G: the existential quantification is involved instead of universal one. 
Let $\mathbf{G}_{0}$ be a first-order formula (in $\mathrm{FO}^{2}$ ) expressing the fact that, for any $\left\langle k, k^{\prime}\right\rangle \in\left\{0, \ldots, 2^{n}-1\right\} \times\{0, \ldots, l\}, \mathrm{R}_{k, k^{\prime}}$, is interpreted as a reflexive and symmetric binary relation. Let $\mathrm{G}_{1}$ be a first-order formula expressing the fact that, for any $i \in\{1, \ldots, q\}, Q_{i}$ is interpreted ${ }^{4}$ as a singleton, e.g.

$$
\bigwedge_{i=1}^{q} \exists \mathrm{y}_{0}\left(\mathrm{Q}_{i}\left(\mathrm{y}_{0}\right) \wedge \forall \mathrm{y}_{1} \neg \mathrm{y}_{0}=\mathrm{y}_{1} \Rightarrow \neg \mathrm{Q}_{i}\left(\mathrm{y}_{1}\right)\right)
$$

In the case when $\operatorname{VARE}(\mathrm{F})=\emptyset, \mathrm{G}_{1} \stackrel{\text { def }}{=} \forall \mathrm{y}_{0} \mathrm{y}_{0}=\mathrm{y}_{0}$. Let $\mathrm{T}_{1}(\mathrm{~F})$ be the first-order formula (in $\mathrm{FO}^{2}[=]$ ) defined by

$$
\mathrm{T}_{1}(\mathrm{~F}) \stackrel{\text { def }}{=} \mathrm{G}_{0} \wedge \mathrm{G}_{1} \wedge \exists \mathrm{y}_{0} S T^{\prime}\left(\mathrm{F}, \mathrm{y}_{0}\right)
$$

The translation is not quite finished yet. Indeed, although at least one of the components $p_{1} \cap P_{1}$ or $p_{1} \cap-P_{1}$ is interpreted by the empty set of properties, this fact is not taken into account in $S T^{\prime}$ (considering e.g. $n=1$ ). This is a serious gap since at least one of the predicate symbols $R_{0,1}$ or $R_{1,1}$ should be interpreted as the universal relation. The forthcoming developments provide an answer to this technical problem.

Let $\mathrm{G}$ be a first-order formula, $k^{\prime} \in\{1, \ldots, l\}$ and $X_{k^{\prime}} \in$ setocc $_{k^{\prime}}$. We write $\mathrm{G}\left[k^{\prime}, X_{k^{\prime}}\right]$ to denote the first-order formula obtained from $\mathrm{G}$ by substituting:

- every occurrence of $\mathrm{R}_{k, k^{\prime}}\left(\mathbf{z}_{1}, \mathbf{z}_{2}\right) \Rightarrow \mathrm{H}$ with $\mathrm{H}$ if $k \in X_{k^{\prime}}$,

- every occurrence of $\mathrm{F}^{\prime} \wedge \mathrm{R}_{k, k^{\prime}}\left(\mathbf{z}_{1}, \mathbf{z}_{2}\right) \wedge \mathrm{F}^{\prime \prime} \Rightarrow \mathrm{H}$ with $\mathrm{F}^{\prime} \wedge \mathrm{F}^{\prime \prime} \Rightarrow \mathrm{H}$ if $k \in X_{k^{\prime}}$ -the degenerate cases are omitted here-

(this rewriting procedure is confluent and always terminates). From a semantical viewpoint, the substitution is equivalent ${ }^{5}$ to satisfaction of the condition $(k \in$ $\left.X_{k^{\prime}}\right): \forall \mathbf{z}_{1}, \mathbf{z}_{2}, \mathrm{R}_{k, k^{\prime}}\left(\mathbf{z}_{1}, \mathbf{z}_{2}\right)$. For $\left\langle X_{1}, \ldots, X_{l}\right\rangle \in$ setocc $_{1} \times \ldots \times$ setoccl $_{l}$, we write $\mathrm{G}\left[X_{1}, \ldots, X_{l}\right]$ to denote the first-order formula $\mathrm{G}\left[1, X_{1}\right]\left[2, X_{2}\right] \ldots\left[l, X_{l}\right]$. Observe that for any permutation $\sigma$ on $\{1, \ldots, l\}$,

$$
\mathrm{G}\left[\sigma(1), X_{\sigma(1)}\right]\left[\sigma(2), X_{\sigma(2)}\right] \ldots\left[\sigma(l), X_{\sigma(l)}\right]=\mathrm{G}\left[1, X_{1}\right]\left[2, X_{2}\right] \ldots\left[l, X_{l}\right]
$$

Let $\mathrm{T}(\mathrm{F})$ be the formula

$$
\mathrm{T}(\mathrm{F}) \stackrel{\text { def }}{=} \bigvee\left\{\mathrm{T}_{1}(\mathrm{~F})\left[X_{1}, \ldots, X_{l}\right]:\left\langle X_{1}, \ldots, X_{l}\right\rangle \in \text { setocc }_{1} \times \ldots \times \text { setocc }_{l}\right\}
$$

Observe that $\mathrm{T}$ is exponential-time in $|\mathrm{F}|$ and the size of the formula obtained by translation may increase exponentially. It is however, not clear whether there

${ }^{4}$ Let $\mathrm{FO}^{2}\left[\exists^{=1}\right]$ be $\mathrm{FO}^{2}$ augmented with the existential quantifier $\exists^{=1}$ meaning "there exists exactly one". $\mathrm{FO}^{2}\left[\exists^{=1}\right.$-satisfiability has been proved to be in NEXPTIME (see e.g. [PST97]). By defining $\mathrm{G}_{1}$ by $\mathrm{G}_{1} \stackrel{\text { def }}{=} \bigwedge_{i=1}^{q} \exists^{=1} \mathrm{y}_{0} \mathrm{Q}_{i}\left(\mathrm{y}_{0}\right)$ we are able to prove decidability of $\mathcal{L}$-satisfiability via a translation into $\mathrm{FO}^{2}\left[\exists^{=1}\right]$.

${ }^{5}$ Another solution consists in defining $\mathrm{G}\left[k^{\prime}, X_{k^{\prime}}\right]$ as the formula $\left(\bigwedge_{k \in X_{k^{\prime}}} \forall \mathrm{y}_{0}, \mathrm{y}_{1}, \mathrm{R}_{k, k^{\prime}}\left(\mathrm{y}_{0}, \mathrm{y}_{1}\right)\right) \wedge \mathrm{G}$ 
exists a tighter translation that characterizes more accurately the complexity class of $\mathcal{L}$-satisfiability. Observe also that $\mathrm{T}(\mathrm{F})$ is classically equivalent to

$\mathrm{G}_{0} \wedge \mathrm{G}_{1} \wedge \exists \mathrm{y}_{0} \bigvee\left\{S T^{\prime}\left(\mathrm{F}, \mathrm{y}_{0}\right)\left[X_{1}, \ldots, X_{l}\right]:\left\langle X_{1}, \ldots, X_{l}\right\rangle \in\right.$ setocc $_{1} \times \ldots \times$ setocc $\left._{l}\right\}$

Example 1. Let $\mathrm{F}$ be the formula $\left\langle\mathrm{p}_{1} \cup \mathrm{p}_{2}\right\rangle \neg \mathrm{E}_{1} \wedge\left[\mathrm{P}_{1}\right] \mathrm{E}_{1}$. Then $\mathrm{F}$ is $\mathcal{L}$-satisfiable, and the translation of $\mathrm{F}$ is the disjunction of the following formulae:

1. $\mathrm{G}_{0} \wedge \mathrm{G}_{1} \wedge \exists \mathrm{y}_{0}\left(\exists \mathrm{y}_{1} \mathrm{R}_{1,1}\left(\mathrm{y}_{0}, \mathrm{y}_{1}\right) \wedge \mathrm{R}_{1,2}\left(\mathrm{y}_{0}, \mathrm{y}_{1}\right) \wedge \neg \mathrm{P}_{1}\left(\mathrm{y}_{1}\right)\right) \wedge\left(\forall \mathrm{y}_{1} \mathrm{R}_{0,0}\left(\mathrm{y}_{0}, \mathrm{y}_{1}\right) \Rightarrow\right.$ $\left.\mathrm{P}_{1}\left(\mathrm{y}_{1}\right)\right)$

2. $\mathrm{G}_{0} \wedge \mathrm{G}_{1} \wedge \exists \mathrm{y}_{0}\left(\exists \mathrm{y}_{1} \mathrm{R}_{0,1}\left(\mathrm{y}_{0}, \mathrm{y}_{1}\right) \wedge \mathrm{R}_{1,2}\left(\mathrm{y}_{0}, \mathrm{y}_{1}\right) \wedge \neg \mathrm{P}_{1}\left(\mathrm{y}_{1}\right)\right) \wedge\left(\forall \mathrm{y}_{1} \mathrm{R}_{0,0}\left(\mathrm{y}_{0}, \mathrm{y}_{1}\right) \wedge\right.$ $\left.\mathrm{R}_{0,1}\left(\mathrm{y}_{0}, \mathrm{y}_{1}\right) \Rightarrow \mathrm{P}_{1}\left(\mathrm{y}_{1}\right)\right)$

3. $\mathrm{G}_{0} \wedge \mathrm{G}_{1} \wedge \exists \mathrm{y}_{0}\left(\exists \mathrm{y}_{1} \mathrm{R}_{1,1}\left(\mathrm{y}_{0}, \mathrm{y}_{1}\right) \wedge \mathrm{R}_{0,2}\left(\mathrm{y}_{0}, \mathrm{y}_{1}\right) \wedge \neg \mathrm{P}_{1}\left(\mathrm{y}_{1}\right)\right) \wedge\left(\forall \mathrm{y}_{1} \mathrm{R}_{0,0}\left(\mathrm{y}_{0}, \mathrm{y}_{1}\right) \wedge\right.$ $\left.\mathrm{R}_{0,2}\left(\mathrm{y}_{0}, \mathrm{y}_{1}\right) \Rightarrow \mathrm{P}_{1}\left(\mathrm{y}_{1}\right)\right)$

4. $\mathrm{G}_{0} \wedge \mathrm{G}_{1} \wedge \exists \mathrm{y}_{0}\left(\exists \mathrm{y}_{1} \mathrm{R}_{0,1}\left(\mathrm{y}_{0}, \mathrm{y}_{1}\right) \wedge \mathrm{R}_{0,2}\left(\mathrm{y}_{0}, \mathrm{y}_{1}\right) \wedge \neg \mathrm{P}_{1}\left(\mathrm{y}_{1}\right)\right) \wedge\left(\forall \mathrm{y}_{1} \mathrm{R}_{0,0}\left(\mathrm{y}_{0}, \mathrm{y}_{1}\right) \wedge\right.$ $\left.\mathrm{R}_{0,1}\left(\mathrm{y}_{0}, \mathrm{y}_{1}\right) \wedge \mathrm{R}_{0,2}\left(\mathrm{y}_{0}, \mathrm{y}_{1}\right) \Rightarrow \mathrm{P}_{1}\left(\mathrm{y}_{1}\right)\right)$

The translation takes into account that $N\left(\mathrm{p}_{1} \cup \mathrm{p}_{2}\right)=\mathrm{A}_{0,1} \cup \mathrm{A}_{1,1} \cup \mathrm{A}_{0,2} \cup \mathrm{A}_{1,2}$ and $N\left(\mathrm{P}_{1}\right)=\mathrm{A}_{0,0} \cup \mathrm{A}_{0,1} \cup \mathrm{A}_{0,2}$.

\subsection{Faithfulness of the translation}

The rest of this section is devoted to proving Proposition 5 below and stating certain corollaries (some being consequences of the proof of Proposition 5).

Proposition 5. (1) F is $\mathcal{L}$-satisfiable iff (2) $\mathrm{T}(\mathrm{F})$ is first-order satisfiable.

Proof. (1) implies (2). First assume $\mathcal{U}, e_{0} \vDash \mathrm{F}$ for some model

$$
\mathcal{U}=\left(E N T, P R O P,\left(\operatorname{sim}_{P}\right)_{P \subseteq P R O P}, v\right)
$$

and $e_{0} \in E N T$ (this is the easier part of the proof). Let us define the following first-order structure $\mathcal{M} \stackrel{\text { def }}{=}\langle D, m\rangle$ :

- $D \stackrel{\text { def }}{=} E N T$; for any $i \in \omega, m\left(\mathbf{Q}_{i}\right) \stackrel{\text { def }}{=} v\left(\mathbf{x}_{i}\right)$ and $m\left(\mathrm{P}_{i}\right) \stackrel{\text { def }}{=} v\left(\mathrm{E}_{i}\right)$;

- for any $\left\langle k, k^{\prime}\right\rangle \in\left\{0, \ldots, 2^{n}-1\right\} \times\{1, \ldots, l\}, m\left(\mathrm{R}_{k, k^{\prime}}\right) \stackrel{\text { def }}{=} \operatorname{sim}_{v\left(\mathrm{~A}_{k, k^{\prime}}\right)}$ (for the other values of $\left\langle k, k^{\prime}\right\rangle$ the interpretation of $\mathrm{R}_{k, k^{\prime}}$ is not constrained).

Let $\left\langle i_{1}, \ldots, i_{l}\right\rangle \in\left\{0, \ldots, 2^{n}-1\right\}^{l}$ be such that for any $k^{\prime} \in\{1, \ldots, l\}$, $v\left(\mathrm{~A}_{i_{k^{\prime}}, k^{\prime}}\right)=v\left(\mathrm{p}_{k^{\prime}}\right)$. Such a sequence $\left\langle i_{1}, \ldots, i_{l}\right\rangle$ is unique. So, for any $k^{\prime} \in$ $\{1, \ldots, l\}, X_{k^{\prime}} \stackrel{\text { def }}{=} o c_{k^{\prime}} \backslash\left\{i_{k^{\prime}}\right\}$. It is easy to show that $\mathcal{M} \models \mathrm{G}_{0} \wedge \mathrm{G}_{1}$ since $\mathcal{U}$ is a model. We claim that $\mathcal{M} \models \mathrm{T}_{1}(\mathrm{~F})\left[X_{1}, \ldots, X_{l}\right]$, and therefore $\mathcal{M} \models \mathrm{T}(\mathrm{F})$. To prove such a result, let us show that for any $\mathrm{G} \in \operatorname{sub}(\mathrm{F}), e \in E N T, i \in\{0,1\}$, $\mathcal{U}, e \vDash \mathrm{G}$ iff $\mathcal{M}, v_{\mathcal{M}}\left[\mathrm{y}_{i} \leftarrow e\right] \models S T^{\prime}\left(\mathrm{G}, \mathrm{y}_{i}\right)\left[X_{1}, \ldots, X_{l}\right]$. We write $v_{\mathcal{M}}\left[\mathrm{y}_{i} \leftarrow e\right]$ to denote a first-order valuation $v_{\mathcal{M}}$ such that $v_{\mathcal{M}}\left(\mathrm{y}_{i}\right)=e$. It entails $\mathcal{M} \models$ $\exists \mathrm{y}_{0} S T^{\prime}\left(\mathrm{G}, \mathrm{y}_{0}\right)\left[X_{1}, \ldots, X_{l}\right]$. We omit the base case and the cases in the induction step when the outermost connective is Boolean. Here are the remaining cases.

Case 1: $\mathrm{G}=[\mathrm{A}] \mathrm{F}_{1}$ and $N(\mathrm{~A})=0$ 
$\mathcal{U}, e \vDash[\mathrm{A}] \mathrm{F}_{1}$ iff for any $e^{\prime} \in \operatorname{sim}_{v(\mathrm{~A})}(e), \mathcal{U}, e^{\prime} \vDash \mathrm{F}_{1}$

iff for any $e^{\prime} \in E N T, \mathcal{U}, e^{\prime} \vDash \mathrm{F}_{1}$

iff for any $e^{\prime} \in D, \mathcal{M}, v_{\mathcal{M}}\left[\mathrm{y}_{i} \leftarrow e^{\prime}\right] \models S T^{\prime}\left(\mathrm{F}_{1}, \mathrm{y}_{i}\right)\left[X_{1}, \ldots, X_{l}\right]$

iff $\mathcal{M} \models \forall \mathrm{y}_{0} S T^{\prime}\left(\mathrm{F}_{1}, \mathrm{y}_{0}\right)\left[X_{1}, \ldots, X_{l}\right]$

iff $\mathcal{M} \vDash S T^{\prime}\left([\mathrm{A}] \mathrm{F}_{1}, \mathrm{y}_{i}\right)\left[X_{1}, \ldots, X_{l}\right]$

Case 2: $\mathrm{G}=[\mathrm{A}] \mathrm{F}_{1}$ and $N(\mathrm{~A})=\mathrm{A}_{k_{1}, k_{1}^{\prime}} \cup \ldots \cup \mathrm{A}_{k_{u}, k_{u}^{\prime}}$

First observe that for any $k^{\prime} \in\{1, \ldots, l\}$ and $k \in X_{k^{\prime}}, v\left(\mathrm{~A}_{k, k^{\prime}}\right)=\emptyset$.

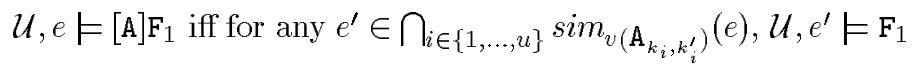

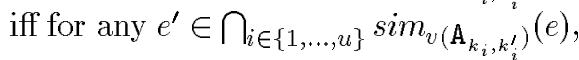

$\mathcal{M}, v_{\mathcal{M}}\left[\mathrm{y}_{1-i} \leftarrow e^{\prime}\right] \vDash S T^{\prime}\left(\mathrm{F}_{1}, \mathrm{y}_{1-i}\right)\left[X_{1}, \ldots, X_{l}\right]$

iff for any $e^{\prime} \in \bigcap_{i \in\{1, \ldots, u\}} m\left(\mathrm{R}_{k_{i}, k_{i}^{\prime}}\right)(e)$,

$\mathcal{M}, v_{\mathcal{M}}\left[\mathrm{y}_{1-i} \leftarrow e^{\prime}\right] \models S T^{\prime}\left(\mathrm{F}_{1}, \mathrm{y}_{1-i}\right)\left[X_{1}, \ldots, X_{l}\right]$

iff $\mathcal{M}, v_{\mathcal{M}}\left[\mathrm{y}_{i} \leftarrow e\right] \vDash \forall \mathrm{y}_{1-i}\left(\mathrm{R}_{k_{1}, k_{1}^{\prime}}\left(\mathrm{y}_{i}, \mathrm{y}_{1-i}\right) \wedge \ldots \wedge \mathrm{R}_{k_{u}, k_{u}^{\prime}}\left(\mathrm{y}_{i}, \mathrm{y}_{1-i}\right)\right) \Rightarrow$ $S T^{\prime}\left(\mathrm{F}_{1}, \mathrm{y}_{1-i}\right)\left[X_{1}, \ldots, X_{l}\right]$

iff $\mathcal{M}, v_{\mathcal{M}}\left[\mathrm{y}_{i} \leftarrow e\right] \vDash \forall \mathrm{y}_{1-i}\left(\bigwedge_{1 \leq i \leq u, k_{i} \notin X_{k_{i}^{\prime}}} \mathrm{R}_{k_{i}, k_{i}^{\prime}}\left(\mathrm{y}_{i}, \mathrm{y}_{1-i}\right)\right) \Rightarrow$ $S T^{\prime}\left(\mathrm{F}_{1}, \mathrm{y}_{1-i}\right)\left[X_{1}, \ldots, X_{l}\right]$

( $\bigwedge_{1 \leq i \leq u, k_{i} \notin X_{k_{i}^{\prime}}} \mathrm{R}_{k_{i}, k_{i}^{\prime}}\left(\mathrm{y}_{i}, \mathrm{y}_{1-i}\right)$ is $\mathrm{T}$ if the conjunction is empty)

iff $^{2} \mathcal{M}, v_{\mathcal{M}}\left[\mathrm{y}_{i} \leftarrow e\right] \vDash\left(\forall \mathrm{y}_{1-i}\left(\mathrm{R}_{k_{1}, k_{1}^{\prime}}\left(\mathrm{y}_{i}, \mathrm{y}_{1-i}\right) \wedge \ldots \wedge \mathrm{R}_{k_{u}, k_{u}^{\prime}}\left(\mathrm{y}_{i}, \mathrm{y}_{1-i}\right)\right) \Rightarrow\right.$ $\left.S T^{\prime}\left(\mathrm{F}_{1}, \mathrm{y}_{1-i}\right)\right)\left[X_{1}, \ldots, X_{l}\right]$

iff $\mathcal{M} \vDash S T^{\prime}\left([\mathrm{A}] \mathrm{F}_{1}, \mathrm{y}_{i}\right)\left[X_{1}, \ldots, X_{l}\right]$

In the previous line the substitution operation is performed only on $S T^{\prime}\left(\mathrm{F}_{1}, \mathrm{y}_{1-i}\right)$ whereas in the next line it is performed on the whole expression.

(2) implies (1). Omitted because of lack of space.

Corollary 6. (1) The $\mathcal{L}$-satisfiability problem is decidable. (2) $\mathcal{L}$ has the finite model property. In particular, every $\mathcal{L}$-satisfiable formula $\mathrm{F}$ has a model such that $\operatorname{card}(E N T) \leq 2^{\left.2^{p(1} \mathbb{F}_{1}\right)}$ for some fixed polynomial $p(n)$, and the cardinality of the relevant part of $\mathcal{U}$ is at most $2^{n}+l$, where $n=\operatorname{card}(\operatorname{VARSP}(\mathrm{F}))$ and $l=\operatorname{card}(\operatorname{VARP}(\mathrm{F}))$.

As observed by one referee, formalizing concepts from similarity theory directly in first-order logic could be another alternative.

\subsection{The degenerate cases}

In the previous section we have assumed that $n \geq 1$ and $l \geq 1$. Now let us examine the remaining cases. If $l \geq 1$ and no variable for sets of properties occurs in the formula $(n=0)$, then we consider the following components: $\mathrm{A}_{0}=-\mathrm{p}_{1} \cap \ldots \cap-\mathrm{p}_{l}$ and $\mathrm{A}_{k^{\prime}}=\mathrm{p}_{k^{\prime}}$ for $k^{\prime} \in\{1, \ldots, l\}$. Condition (3) in the definition of $S T^{\prime}$ becomes:

$$
S T^{\prime}\left([\mathrm{A}] \mathrm{G}, \mathrm{y}_{i}\right) \stackrel{\text { def }}{=}\left\{\begin{array}{l}
\forall \mathrm{y}_{0} S T^{\prime}\left(\mathrm{G}, \mathrm{y}_{0}\right) \text { if } N(\mathrm{~A})=0 \\
\forall \mathrm{y}_{1-i}\left(\mathrm{R}_{0, k_{1}^{\prime}}\left(\mathrm{y}_{i}, \mathrm{y}_{1-i}\right) \wedge \ldots \wedge \mathrm{R}_{0, k_{u}^{\prime}}\left(\mathrm{y}_{i}, \mathrm{y}_{1-i}\right)\right) \Rightarrow S T^{\prime}\left(\mathrm{G}, \mathrm{y}_{1-i}\right) \\
\text { if } N(\mathrm{~A})=\mathrm{A}_{k_{1}^{\prime}} \cup \ldots \cup \mathrm{A}_{k_{u}^{\prime}}
\end{array}\right.
$$


If $n \geq 1$ and no variable for individual properties occurs in the formula $(l=0)$, then Condition (3) in the definition of $S T^{\prime}$ becomes:

$$
S T^{\prime}\left([\mathrm{A}] \mathrm{G}, \mathrm{y}_{i}\right) \stackrel{\text { def }}{=}\left\{\begin{array}{l}
\forall \mathrm{y}_{0} S T^{\prime}\left(\mathrm{G}, \mathrm{y}_{0}\right) \text { if } N(\mathrm{~A})=0 \\
\forall \mathrm{y}_{1-i}\left(\mathrm{R}_{k_{1}, 0}\left(\mathrm{y}_{i}, \mathrm{y}_{1-i}\right) \wedge \ldots \wedge \mathrm{R}_{k_{u}, 0}\left(\mathrm{y}_{i}, \mathrm{y}_{1-i}\right)\right) \Rightarrow S T^{\prime}\left(\mathrm{G}, \mathrm{y}_{1-i}\right) \\
\text { if } N(\mathrm{~A})=\mathrm{B}_{k_{1}} \cup \ldots \cup \mathrm{B}_{k_{u}} \text { (see Section 3.2) }
\end{array}\right.
$$

Moreover, $\mathrm{T}(\mathrm{F})$ is simply defined as $\mathrm{T}_{1}(\mathrm{~F})$. In the case when $n=0, l=0$ and $\operatorname{TERM}(\mathrm{F}) \neq \emptyset$, by substituting every occurrence of 0 in $\mathrm{F}$ by $\mathrm{p}_{1} \cap-\mathrm{p}_{1}$ we preserve $\mathcal{L}$-satisfiability and reduce the case to the previous one. Otherwise, $\mathrm{F}$ is a formula of the propositional calculus and therefore it poses no difficulty with respect to decidability.

\section{Decidability results for variants of $\mathcal{L}$}

\subsection{Fixed finite set of properties}

In this section we consider a finite set $P R O P$ of properties, and show that $\mathcal{L}_{P R O P}$ shares various features with $\mathcal{L}$. Actually $\mathcal{L}_{P R O P}$ corresponds to the similarity logic with a fixed finite set of properties defined in [Kon97]. Without any loss of generality we can assume that $P R O P=\{1, \ldots, \alpha\}$ for some $\alpha \geq 1$ and $\operatorname{VARP}=\left\{\mathrm{p}_{1}, \ldots, \mathrm{p}_{\alpha}\right\}$.

Let $\mathrm{F}$ be a $\mathcal{L}_{P R O P}$-formula such that $\operatorname{VARP}(\mathrm{F})=\left\{\mathrm{p}_{i_{1}}, \ldots, \mathrm{p}_{i_{l}}\right\}$ and $\operatorname{VARSP}(\mathrm{F})=$ $\left\{\mathrm{P}_{j_{1}}, \ldots, \mathrm{P}_{j_{n}}\right\}$. For any interpretation $v$ possibly occurring in some $\mathcal{L}_{P R O P}$-model and for any $\mathrm{A} \in \mathrm{TERM}(\mathrm{F})$, if $v(\mathrm{~A})=\left\{k_{1}, \ldots, k_{s}\right\}$, then $N_{v}(\mathrm{~A}) \stackrel{\text { def }}{=} \mathrm{p}_{k_{1}} \cup \ldots \cup \mathrm{p}_{k_{s}}$ otherwise $(v(\mathrm{~A})=\emptyset) N_{v}(\mathrm{~A}) \stackrel{\text { def }}{=} 0$. We write $v_{\mathrm{F}}$ (resp. $N_{v}(\mathrm{~F})$ ) to denote the restriction of $v$ to TERM $(\mathrm{F})$ (resp. the formula obtained from $\mathrm{F}$ by substituting every occurrence of A by $\left.N_{v}(\mathrm{~A})\right)$. Let $X_{\mathrm{F}}$ be the finite set

$$
\left\{v_{\mathrm{F}}: v \text { interpretation possibly occurring in some } \mathcal{L}_{P R O P}-\text { model }\right\}
$$

Proposition 7. Let $\mathrm{F}$ be a $\mathcal{L}_{P R O P}$-formula. (1) $\mathrm{F}$ is $\mathcal{L}_{P R O P}$-satisfiable iff (Q) $\bigvee_{v^{\prime \prime} \in X_{\mathrm{F}}} N_{v^{\prime \prime}}(\mathrm{F})$ is $\mathcal{L}$-satisfiable.

Proof. (1) implies (2): Assume $\mathcal{U}=\left(E N T, P R O P,\left(\operatorname{sim}_{P}\right)_{P \subset P R O P}, v\right), e_{0} \models \mathrm{F}$ for some $e_{0} \in E N T$. It is easy to check that $\mathcal{U}^{\prime}, e_{0} \vDash N_{v}(\mathrm{~F})$ where $\mathcal{U}^{\prime}$ is defined from $\mathcal{U}$ by only replacing $v$ by $v^{\prime}$ defined as follows: for any $i \in\{1, \ldots, \alpha\}$, $v^{\prime}\left(\mathrm{p}_{i}\right) \stackrel{\text { def }}{=}\{i\}$. Let $\mathcal{U}^{\prime \prime}=\left(E N T, \omega,\left(\operatorname{sim}_{P}^{\prime \prime}\right)_{P \subseteq \omega}, v^{\prime \prime}\right)$ be an $\mathcal{L}$-model such that

- $v^{\prime}$ and $v^{\prime \prime}$ are identical for the common sublanguage,

- for any $i>\alpha, v^{\prime \prime}\left(\mathrm{p}_{i}\right) \stackrel{\text { def }}{=}\{i\}$, for any $P \subseteq \omega, \operatorname{sim}_{P}^{\prime \prime} \stackrel{\text { def }}{=} \operatorname{sim}_{P \cap P R O P}$.

It is a routine task to check that $\mathcal{U}^{\prime \prime}, e_{0} \vDash N_{v}(\mathrm{~F})$ and therefore $\mathcal{U}^{\prime \prime}, e_{0} \models$ $\bigvee_{v^{\prime \prime} \in X_{\mathrm{F}}} N_{v^{\prime \prime}}(\mathrm{F})$. Indeed, for any $\mathrm{A} \in \mathrm{TERM}(\mathrm{F}), \operatorname{sim}_{v(\mathrm{~A})}=\operatorname{sim}_{v^{\prime \prime}\left(N_{v}(\mathrm{~A})\right)^{\prime \prime}}$.

(2) implies (1): Now assume $\bigvee_{v^{\prime \prime} \in X_{\mathrm{F}}} N_{v^{\prime \prime}}(\mathrm{F})$ is $\mathcal{L}$-satisfiable. There exist an $\mathcal{L}$-model $\mathcal{U}^{\prime}=\left(E N T^{\prime}, P R O P^{\prime},\left(\operatorname{sim}_{P}^{\prime}\right)_{P \subseteq P R O P^{\prime}}, v^{\prime}\right), e_{0} \in E N T^{\prime}$ and $v_{0}^{\prime \prime} \in X_{\mathrm{F}}$ 
such that $\mathcal{U}^{\prime}, e_{0} \models N_{v_{0}^{\prime \prime}}(\mathrm{F})$. By the proof of Proposition 5, we can assume that $\left\{u_{1}, \ldots, u_{\alpha}\right\}$ is a relevant part of $P R O P^{\prime}$ in $\mathcal{U}^{\prime}$ such that for any $i \in\{1, \ldots, \alpha\}$, $v^{\prime}\left(\mathrm{p}_{i}\right)=u_{i}$. Indeed, $P R O P^{\prime}$ is at least countable, $\operatorname{VARSP}\left(\bigvee_{v^{\prime \prime} \in X_{\mathrm{F}}} N_{v^{\prime \prime}}(\mathrm{F})\right)=\emptyset$ and $\operatorname{card}\left(\operatorname{VARP}\left(\bigvee_{v^{\prime \prime} \in X_{\mathrm{F}}} N_{v^{\prime \prime}}(\mathrm{F})\right)\right) \leq \alpha$. Let $\mathcal{U}=\left(E N T^{\prime}, P R O P,\left(\operatorname{sim}_{P}\right)_{P \subseteq P R O P}, v\right)$ be the $\mathcal{L}_{P R O P}$-model such that:

- $v$ and $v_{0}^{\prime \prime}$ are identical for the common sublanguage;

- for any $P \subseteq P R O P, \operatorname{sim}_{P} \stackrel{\text { def }}{=} \operatorname{sim}_{\left\{u_{i}: i \in P\right\}}^{\prime}$.

It is a routine task to check that $\mathcal{U}, e_{0} \vDash \mathrm{F}$ since for any $\mathrm{A} \in \mathrm{TERM}(\mathrm{F}), \operatorname{sim}_{v(\mathrm{~A})}=$ $\operatorname{sim}_{v^{\prime}\left(N_{v_{0}^{\prime \prime}}^{\prime}(\mathbf{A})\right)}^{\prime}$

Corollary 8. $\mathcal{L}_{P R O P}$-satisfiability is decidable and $\mathcal{L}_{P R O P}$ has the finite model property.

Example 2. (Example 1 continued) Let $\mathrm{F}$ be the formula $\left\langle\mathrm{p}_{1} \cup \mathrm{p}_{2}\right\rangle \neg \mathrm{E}_{1} \wedge\left[\mathrm{P}_{1}\right] \mathrm{E}_{1}$ for the logic $\mathcal{L}_{\{1,2\}}$. Then, $\mathrm{F}$ is not $\mathcal{L}_{\{1,2\}}$-satisfiable, although $\mathrm{F}$ is $\mathcal{L}$-satisfiable. The formula $\bigvee_{v^{\prime} \in X_{\mathrm{F}}} N_{v^{\prime}}(\mathrm{F})$ is the disjunction of the following formulae:

1. $\left(\left\langle\mathrm{p}_{1} \cup \mathrm{p}_{2}\right\rangle \neg \mathrm{E}_{1} \wedge[0] \mathrm{E}_{1}\right) \vee\left(\left\langle\mathrm{p}_{1} \cup \mathrm{p}_{2}\right\rangle \neg \mathrm{E}_{1} \wedge\left[\mathrm{p}_{1}\right] \mathrm{E}_{1}\right)$

2. $\left(\left\langle\mathrm{p}_{1} \cup \mathrm{p}_{2}\right\rangle \neg \mathrm{E}_{1} \wedge\left[\mathrm{p}_{2}\right] \mathrm{E}_{1}\right) \vee\left(\left\langle\mathrm{p}_{1} \cup \mathrm{p}_{2}\right\rangle \neg \mathrm{E}_{1} \wedge\left[\mathrm{p}_{1} \cup \mathrm{p}_{2}\right] \mathrm{E}_{1}\right)$

\subsection{Fixed infinite set of properties}

In this section we consider some infinite set $P R O P$ of properties, and show that $\mathcal{L}_{P R O P}$ shares various features with $\mathcal{L}$. Actually, $\mathcal{L}_{P R O P}$ corresponds to the similarity logic with a fixed infinite set of properties defined in [Kon97]. Without any loss of generality we can assume that $\omega \subseteq P R O P$ (there is an injective map $f$ from $\omega$ into $P R O P$ ) and $\left\{\mathrm{p}_{1}, \mathrm{p}_{2}, \ldots\right\} \subseteq$ VARP.

Proposition 9. Let $\mathrm{F}$ be a $\mathcal{L}_{P R O P}$-formula. (1) $\mathrm{F}$ is $\mathcal{L}_{P R O P}$-satisfiable iff (D) $\mathrm{F}$ is $\mathcal{L}$-satisfiable.

Proof. Omitted because of lack of space.

Corollary 10. $\mathcal{L}_{P R O P}$-satisfiability is decidable and $\mathcal{L}_{P R O P}$ has the finite model property.

\section{Concluding remarks}

We have shown that the relative similarity logics $\mathcal{L}$ and $\mathcal{L}_{X}$ for some non-empty set $X$ of properties have a decidable satisfiability problems. Moreover, we have also established that such logics have the finite model property. The decidability proof reduces satisfiability in our logic to satisfiability in $\mathrm{FO}^{2}[=]$, a decidable fragment of classical logic [Mor75]. Although our reduction takes advantage of the standard translation ST [Ben83] of modal logic into classical logic, the novelty of our approach consists in the method of handling nominals for atomic properties and decomposition in terms of components encoded in the translation. The reduction into $\mathrm{FO}^{2}[=]$ can be generalized to any relative logics provided, 
1. the conditions ${ }^{6}$ on the relations of the models can be expressed by a firstorder formula involving at most two variables (see the definition of the formula $\mathrm{G}_{0}$ in Section 3.3), and

2. the class of binary relations underlying the logic is closed under intersection.

For instance, if in the definition of $\mathcal{L}$ we replace reflexivity by weak reflexivity, then decidability and finite model property still hold true ${ }^{7}$. This is particularly interesting since weakly reflexive and symmetric modal frames represent exactly the negative similarity relations in information systems (see e.g. [Vak91a, Do96]). For the sake of comparison, the class of reflexive and symmetric modal frames represent precisely the positive similarity relation in information systems.

We have also shown that $\mathcal{L}$-satisfiability is EXPTIME-hard (by taking advantage of the general results from [Hem96]), and that the problem can be solved by a deterministic Turing machine in time $O\left(2^{2^{2^{(n)}}}\right)$ for some polynomial $p(n)$, where $n$ is the length of the tested formula. Indeed: the translation process $\mathrm{T}$ is exponential in time in the length of the formula, $\mathrm{T}$ may increase exponentially the length of the formula and satisfiability for $\mathrm{FO}^{2}[=]$ is in NEXPTIME. It is therefore an open problem to characterize more accurately the complexity class of $\mathcal{L}$-satisfiability. However, the translations we have established can already be used to mechanize the relative similarity logics by taking $\mathrm{FO}^{2}[=]$ as the target logic and by using a theorem prover dedicated to it. We conjecture that more efficient methods might exist for the mechanization.

\section{References}

[Ba197] Ph. Balbiani. Axiomatization of logics based on Kripke models with relative accessibility relations. In [Oe97], pages 553-578, 1997.

[Ben83] J. van Benthem. Modal logic and classical logic. Bibliopolis, 1983.

[Ben98] J. van Benthem. The range of modal logic - An essay in memory of George Gargov. Journal of Applied Non-Classical Logics, 1998. To appear.

[Bla93] P. Blackburn. Nominal tense logic. Notre Dame Journal of Formal Logic, 34(1):56-83, 1993.

[Dem98] S. Demri. A logic with relative knowledge operators. Journal of Logic, Language and Information, 1998. To appear.

[DO96] S. Demri and E. Orłowska. Informational representability: Abstract models versus concrete models. In D. Dubois and H. Prade, editors, Linz Seminar on Fuzzy Sets, Logics and Artificial Intelligence, Linz, Austria. Kluwer Academic Publishers, February 1996. To appear.

[FdCO84] L. Fariñas del Cerro and E. Orłowska. DAL - A logic for data analysis. In T. O'Shea, editor, ECAI-6, pages 285-294, September 1984.

${ }^{6}$ If the conditions require more than two variables, the translation into $\mathrm{FO}^{k}[=]$ for some $k>2$ is also possible. For instance, the logic defined in [Orlo84a] can be translated into $\mathrm{FO}^{3}[=]$ by taking advantage of the results of the present paper.

${ }^{7}$ A binary relation $R$ is weakly reflexive iff for any element $x$ of some domain either $R(x)=\emptyset$ or $(x, x) \in R$. 
[GG93] G. Gargov and V. Goranko. Modal logic with names. Journal of Philosophical Logic, 22(6):607-636, December 1993.

[GKV97] E. Grädel, Ph. Kolaitis, and M. Vardi. On the decision problem for twovariable first-order logic. Bulletin of Symbolic Logic, 3(1):53-69, 1997.

[GP90] G. Gargov and S. Passy. A note on boolean modal logic. In P. Petkov, editor, Summer School and Conference on Mathematical Logic '88, pages 299-309. Plenum Press, 1990.

[Hem96] E. Hemaspaandra. The price of universality. Notre Dame Journal of Formal Logic, 37(2):173-203, 1996.

[Kon97] B. Konikowska. A logic for reasoning about relative similarity. Studia Logica, 58(1):185-226, 1997.

[Lew80] H. Lewis. Complexity results for classes of quanticational formulas. Journal of Computer and System Sciences, 21:317-353, 1980.

[Mor75] M. Mortimer. On language with two variables. Zeit. für Math. Logik and Grund. der Math., 21:135-140, 1975.

[Oe97] E. Orlowska (ed.). Incomplete Information: Rough Set Analysis. Studies in Fuzziness and Soft Computing. Physica-Verlag, Heidelberg, 1997.

[OP84] E. Orlowska and Z. Pawlak. Representation of nondeterministic information. Theoretical Computer Science, 29:27-39, 1984.

[Orło84a] E. Orlowska. Logic of indiscernibility relations. In A. Skowron, editor, 5th Symposium on Computation Theory, Zaborów, Poland, pages 177-186. LNCS 208, Springer-Verlag, 1984.

[Orło84b] E. Orlowska. Modal logics in the theory of information systems. Zeitschr. f. Math. Logik und Grundlagen d. Math., 30(1):213-222, 1984.

[Paw81] Z. Pawlak. Information systems theoretical foundations. Information Systems, 6(3):205-218, 1981.

[PST97] L. Pacholski, W. Szwast, and L. Tendera. Complexity of two-variable logic with counting. In LICS, pages 318-327. IEEE, July 1997.

[PT91] S. Passy and T. Tinchev. An essay in combinatory dynamic logic. Information and Computation, 93:263-332, 1991.

[Vak91a] D. Vakarelov. Logical analysis of positive and negative similarity relations in property systems. In M. de Glas and D. Gabbay, editors, First World Conference on the Fundamentals of Artificial Intelligence, 1991.

[Vak91b] D. Vakarelov. A modal logic for similarity relations in Pawlak knowledge representation systems. Fundamenta Informaticae, 15:61-79, 1991.

[Vak91c] D. Vakarelov. Modal logics for knowledge representation systems. Theoretical Computer Science, 90:433-456, 1991.

[Var97] M. Vardi. Why is modal logic so robustly decidable? In Descriptive complexity and finite models, A.M.S., 1997.

This article was processed using the IATEX macro package with LLNCS style 